\title{
Using Binary Code Instrumentation in Computer Security
}

\author{
Marius POPA ${ }^{1}$, Sergiu CAPISIZU ${ }^{2}$ \\ ${ }^{1}$ Department of Economic Informatics and Cybernetics \\ Bucharest University of Economic Studies, Romania \\ ${ }^{2}$ Bucharest Bar Association \\ marius.popa@ase.ro, capisizu@ew.ro
}

The paper approaches the low-level details of the code generated by compilers whose format permits outside actions. Binary code modifications are manually done when the internal format is known and understood, or automatically by certain tools developed to process the binary code. The binary code instrumentation goals may be various from security increasing and bug fixing to development of malicious software. The paper highlights the binary code instrumentation techniques by code injection to increase the security and reliability of a software application. Also, the paper offers examples for binary code formats understanding and how the binary code injection may be applied.

Keywords: Binary Code, Code Instrumentation, Code Injection

\section{1} Binary Code Formats

The binary code is the output of the compiling process. It uses two binary digits, 0 and 1 , to represent symbols or computer processor instructions as bit strings.

The binary code is directly executed by the computer processor or is interpreted by a specialized software component which translates the binary code into the format understood by the computer processor. Therefore, the binary code is classified into two groups:

- Native code - is generated by the compiler depending on the hardware features; the binary files containing native code are executed only by computer processor units (CPU) having the same features like the computer processor used to generate it; some significant advantages are execution speed and absence of other software components;

- Intermediate code - is generated by the compiler according to intermediate language specifications to be interpreted by a virtual machine; some examples of virtual machines are Java Virtual Machine (JVM) for Java platform and Common Language Runtime (CLR) for .NET framework; a big advantage is the binary code portability for different hardware and software platforms.
Some considerations related to binary code and file formats are presented in [1], [2], [4], [5], [6], [8] and [9].

The memory layout of native code file for a $\mathrm{C}$ program is depicted in figure 1 [11].

The user space is structured into the following segments:

- Code segment - contains executable instructions in binary format; it is a readonly segment and is shared among concurrent users;

- Data segment - contains static and global data initialized in the program code; each process has its own data segment; the data segment is not a executable one;

- Block Started by Symbol (BSS) segment - contains static and global data uninitialized in the program code; it is not an executable segment;

- Stack segment - is used to store local variables (declared inside the functions) and to pass parameters to functions; the stack addresses are allocated from higher memory to lower memory; it is managed by Stack Pointer (SP)/Extended Stack Pointer (ESP)/ Register Stack Pointer (RSP) register;

- Heap segment - is used to allocate memory at running time; heap allocation is managed by operating system; the 
heap addresses are managed by pointer variables.

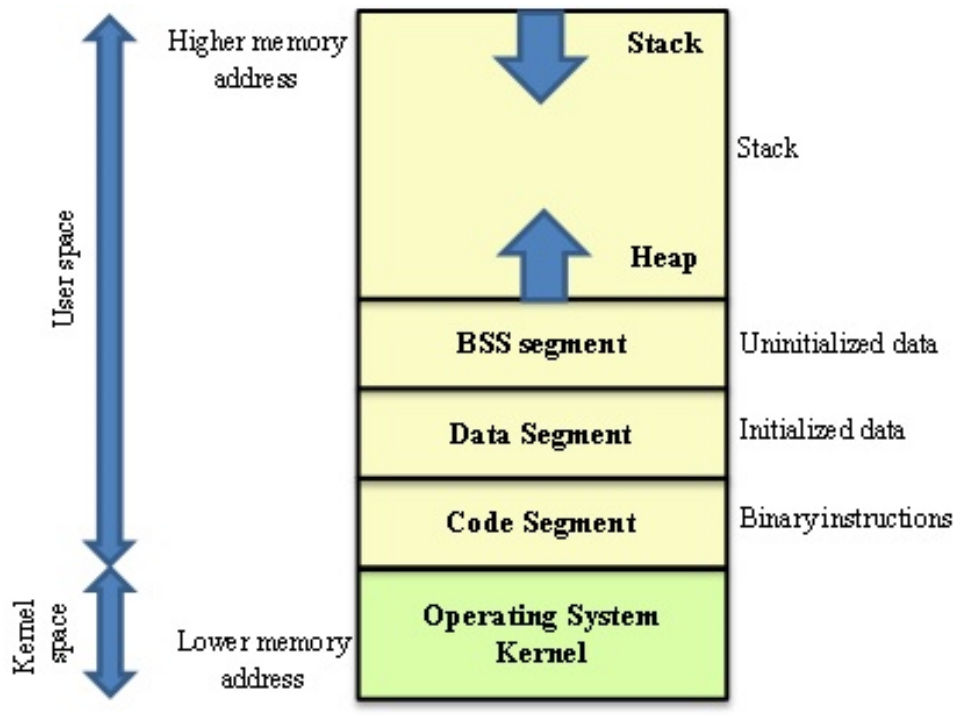

Fig. 1. Memory address space of a process

Segment registers are memory pointers storage or code execution starts. The segment placed inside the computer processor unit. registers for $\mathrm{x} 86$ architecture are presented in They point to memory address where a data Table 1.

Table 1. The segment registers for $\mathrm{x} 86$ computer architecture

\begin{tabular}{|l|l|}
\hline \multicolumn{1}{|c|}{ Name } & \multicolumn{1}{c|}{ Content } \\
\hline CS - Code Segment & Pointer to the code location \\
\hline SS - Stack Segment & Pointer to the stack location \\
\hline DS - Data Segment & Pointer to the data location \\
\hline ES - Extra Segment & Pointer to extra data \\
\hline FS - F Segment & Pointer to extra data \\
\hline GS - G Segment & Pointer to extra data \\
\hline
\end{tabular}

It considers the following $\mathrm{C}++$ program source:

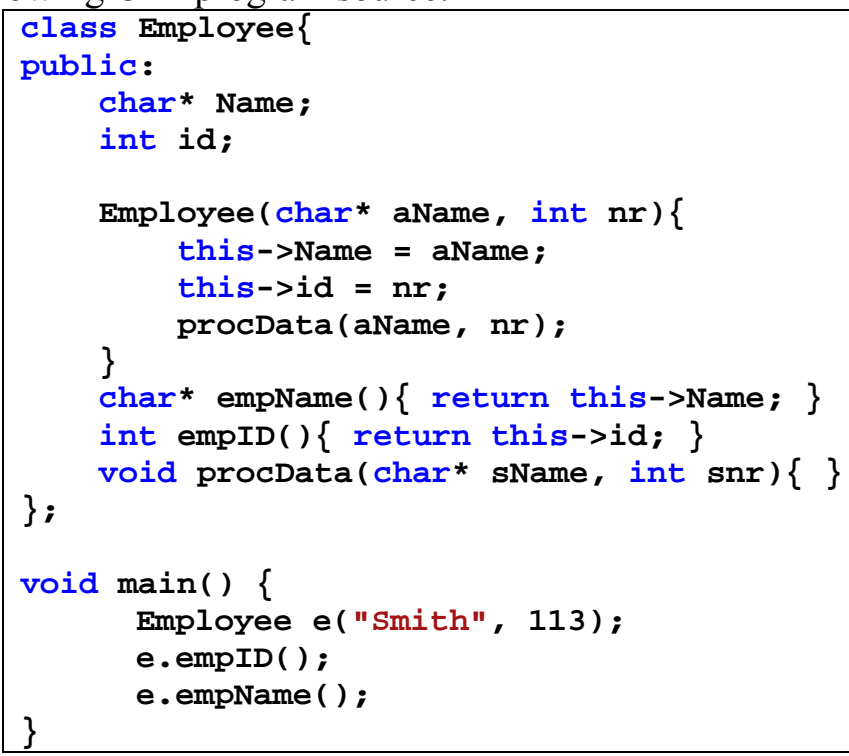


The native code built by Microsoft Visual presented in Table 2.

Studio 2010, x64 version, for each method is

Table 2. Native code for Employee class

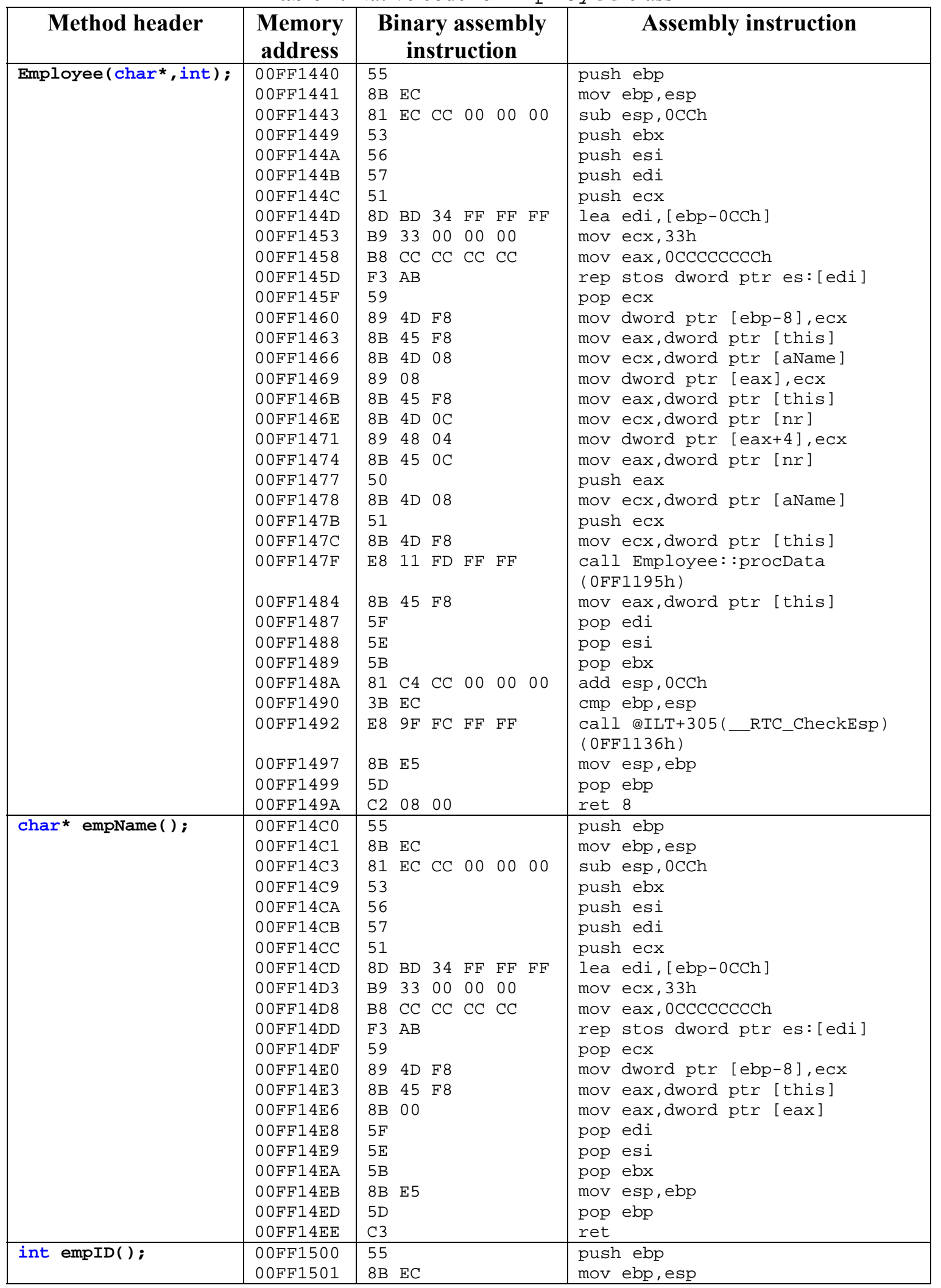




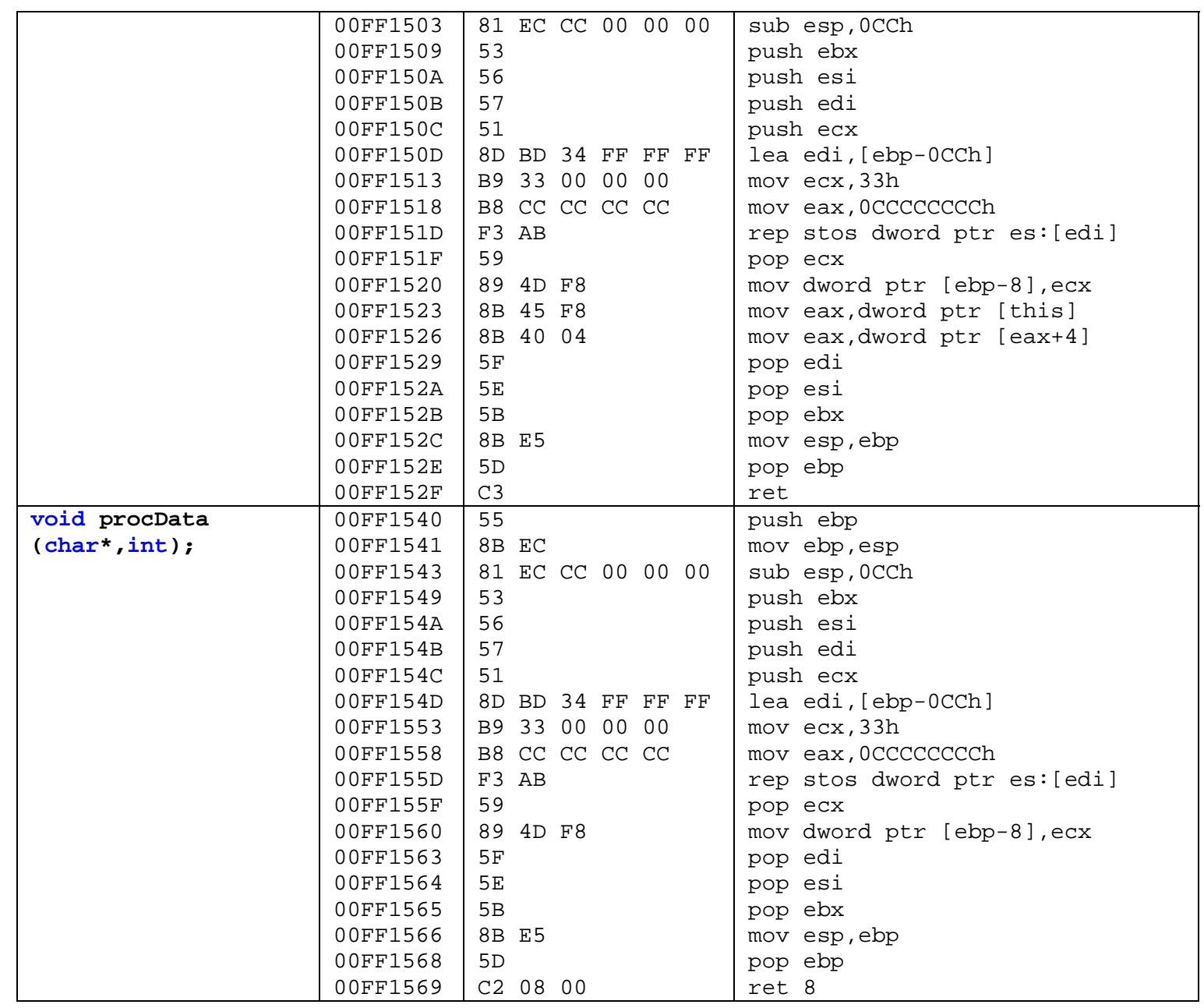

The main function has the following native code content:

Table 3. Native code for main function

\begin{tabular}{|c|c|c|c|}
\hline $\begin{array}{l}\text { Function } \\
\text { header }\end{array}$ & $\begin{array}{l}\text { Memory } \\
\text { address }\end{array}$ & $\begin{array}{c}\text { Binary assembly } \\
\text { instruction }\end{array}$ & Assembly instruction \\
\hline void main(); & 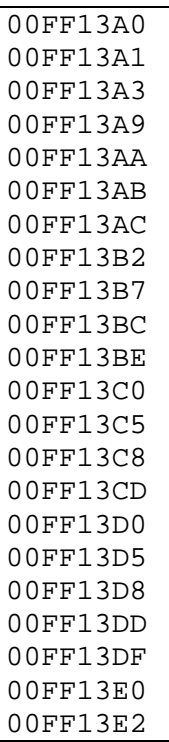 & 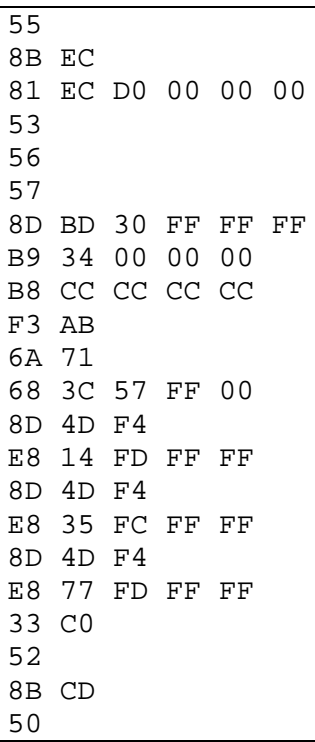 & 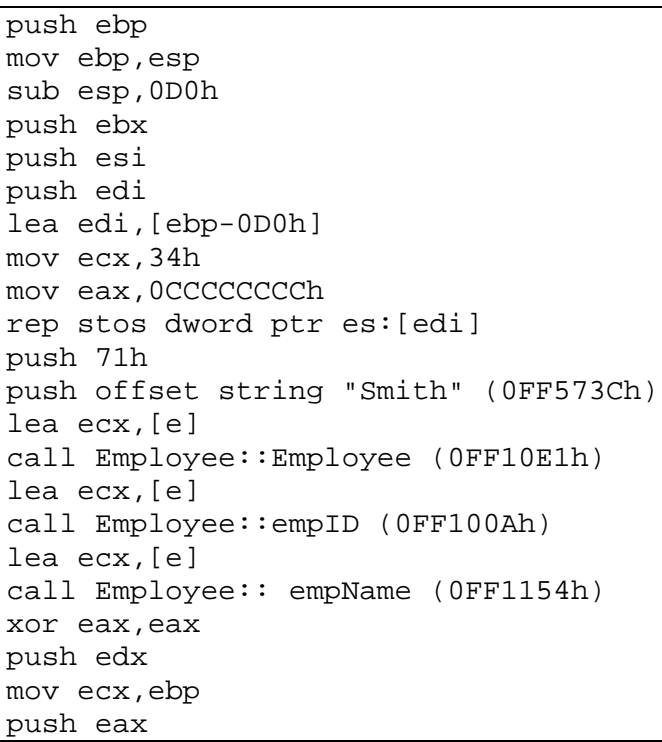 \\
\hline
\end{tabular}




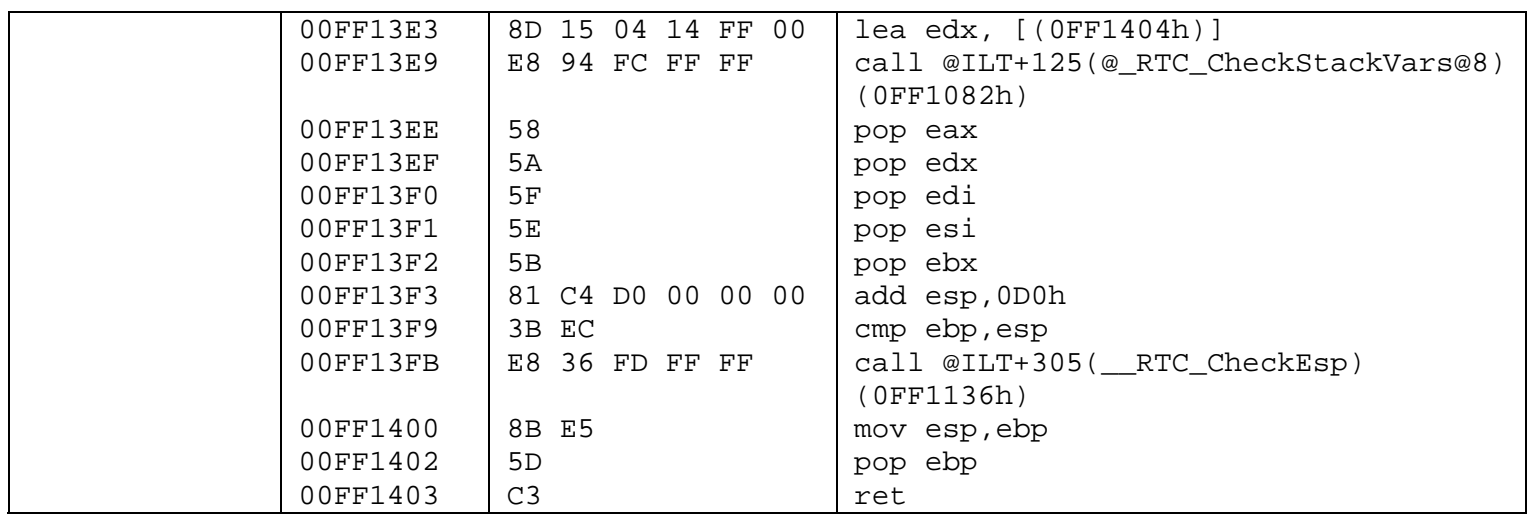

Tables 2 and 3 contain numerical values for memory addresses and binary code in base16 format. The assembly instructions and their binary codes are generated for $\mathrm{x} 86$ computer architecture.

The intermediate code provides the portability feature to a software application. A virtual machine interprets the operation codes from intermediate code and translates them into native code for the machine which runs the virtual machine. The operation codes are associated to intermediate instructions like the native code with the assembly instructions. The intermediate instructions are used within an intermediate language representing the lowest-level human-readable programming language.

For .NET Framework, the intermediate language is called Common Intermediate Language (CIL) and it is defined by Common Language Infrastructure (CLI). The CIL is object-oriented and stack-based. Unlike the CPU architecture, the stack-based architecture pushes data on a stack instead pulling data from registers.

The Employee class and main function defined above have the following content written in $\mathrm{CH}$ programming language:

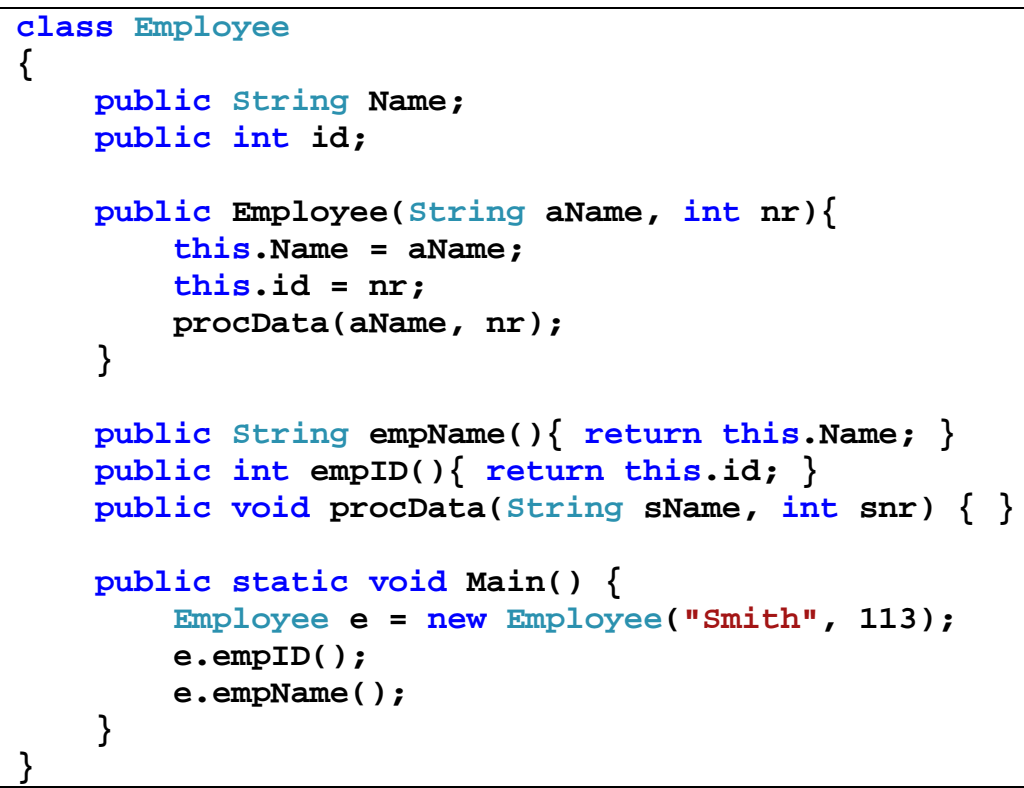

The intermediate code built by Microsoft Visual Studio 2010, x64 version, for each method is presented in Table 4. 
Table 4. C\# .NET Intermediate code for Employee class

\begin{tabular}{|c|c|c|c|}
\hline Method header & $\begin{array}{c}\text { IL } \\
\text { offset }\end{array}$ & $\begin{array}{c}\text { Binary } \\
\text { intermediate } \\
\text { instruction }\end{array}$ & Intermediate instruction \\
\hline $\begin{array}{l}\text { Employee(String, } \\
\text { int); }\end{array}$ & 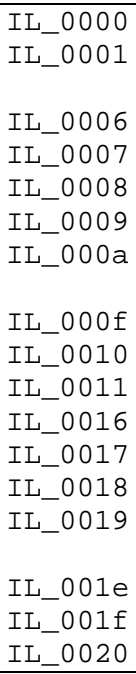 & 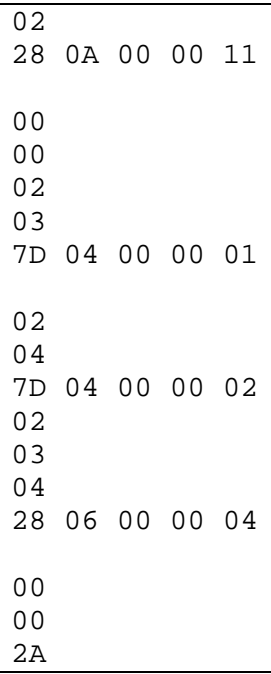 & $\begin{array}{l}\text { ldarg. } 0 \\
\text { call instance void [mscorlib] System. } \\
\text { Object: :.ctor() } \\
\text { nop } \\
\text { nop } \\
\text { ldarg. } \odot \\
\text { ldarg.1 } \\
\text { stfld string EmployeeApp. Employee } \\
\text { : : Name } \\
\text { ldarg. } \odot \\
\text { ldarg.2 } \\
\text { stfld int32 EmployeeApp.Employee: :id } \\
\text { ldarg. } \odot \\
\text { ldarg.1 } \\
\text { ldarg.2 } \\
\text { call instance void EmployeeApp. } \\
\text { Employee: procData(string, int32) } \\
\text { nop } \\
\text { nop } \\
\text { ret }\end{array}$ \\
\hline $\begin{array}{l}\text { String } \\
\text { empName ( ); }\end{array}$ & 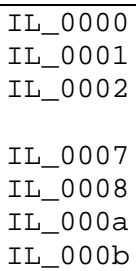 & $\begin{array}{lllll}\odot \odot & & & & \\
\odot 2 & & & & \\
7 B & 04 & 0 \odot & \odot \odot & 01 \\
& & & & \\
\odot A & & & \\
2 B & \odot \odot & & \\
\odot 6 & & & \\
2 A & & & \end{array}$ & 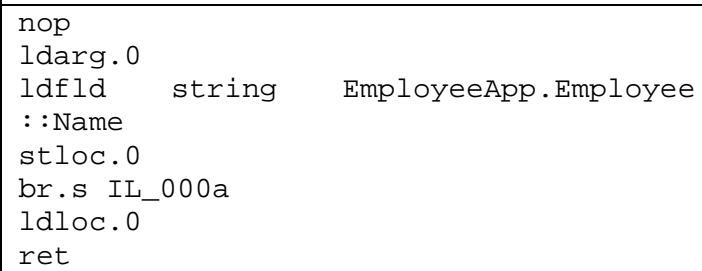 \\
\hline int empID(); & 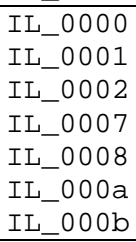 & $\begin{array}{llllll}\odot \odot & & & & \\
\odot 2 & & & & \\
7 B & 04 & 0 \odot & 0 \odot & 02 \\
\odot A & & & & \\
2 B & \odot \odot & & & \\
\odot 6 & & & & \\
2 A & & & & \end{array}$ & 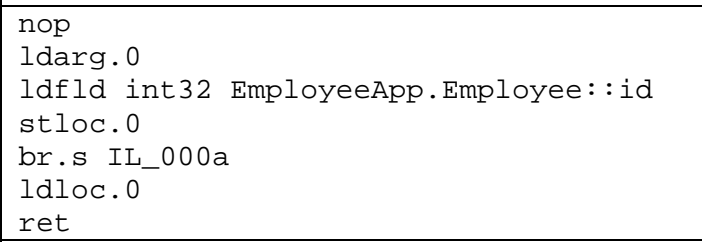 \\
\hline $\begin{array}{l}\text { void procData } \\
\text { (String, int) }\end{array}$ & 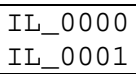 & $\begin{array}{l}\odot \odot \\
2 A\end{array}$ & $\begin{array}{l}\text { nop } \\
\text { ret }\end{array}$ \\
\hline $\begin{array}{l}\text { static void } \\
\text { Main( ); }\end{array}$ & 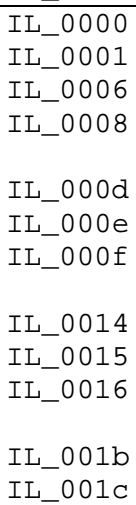 & $\begin{array}{lllll}\odot \odot & & & & \\
72 & 7 \odot & \odot \odot & \odot \odot & \odot 1 \\
1 F & 71 & & & \\
73 & \odot 6 & \odot \odot & \odot \odot & 01 \\
& & & & \\
\odot A & & & & \\
\odot 6 & & & & \\
6 F & \odot 6 & \odot \odot & \odot \odot & \odot 3 \\
& & & & \\
26 & & & & \\
\odot 6 & & & & \\
6 F & \odot 6 & \odot \odot & \odot \odot & \odot 2 \\
& & & & \\
26 & & & & \\
2 A & & & & \end{array}$ & $\begin{array}{l}\text { nop } \\
\text { ldstr "Smith" } \\
\text { ldc.i4.s } 113 \\
\text { newobj instance void EmployeeApp. } \\
\text { Employee: :.ctor(string, int32) } \\
\text { stloc. } 0 \\
\text { ldloc. } \\
\text { callvirt instance int32 EmployeeApp. } \\
\text { Employee: : empID() } \\
\text { pop } \\
\text { ldloc. } \odot \\
\text { callvirt instance string EmployeeApp. } \\
\text { Employee: : empName() } \\
\text { pop } \\
\text { ret }\end{array}$ \\
\hline
\end{tabular}

The intermediate language (IL) offset represents the position of the current intermediate instruction relative to the beginning section where the method intermediate code is stored in the file translated by .NET virtual machine into native code. The intermediate code is organized in accordance with Portable Executable (PE) specifications for .NET applications.

The Java bytecode is the output of a Java compiler containing the binary content of the 
intermediate code. The bytecode files have a In Java programming language, the above specific organization to be interpreted by example has the following content:

JVM.

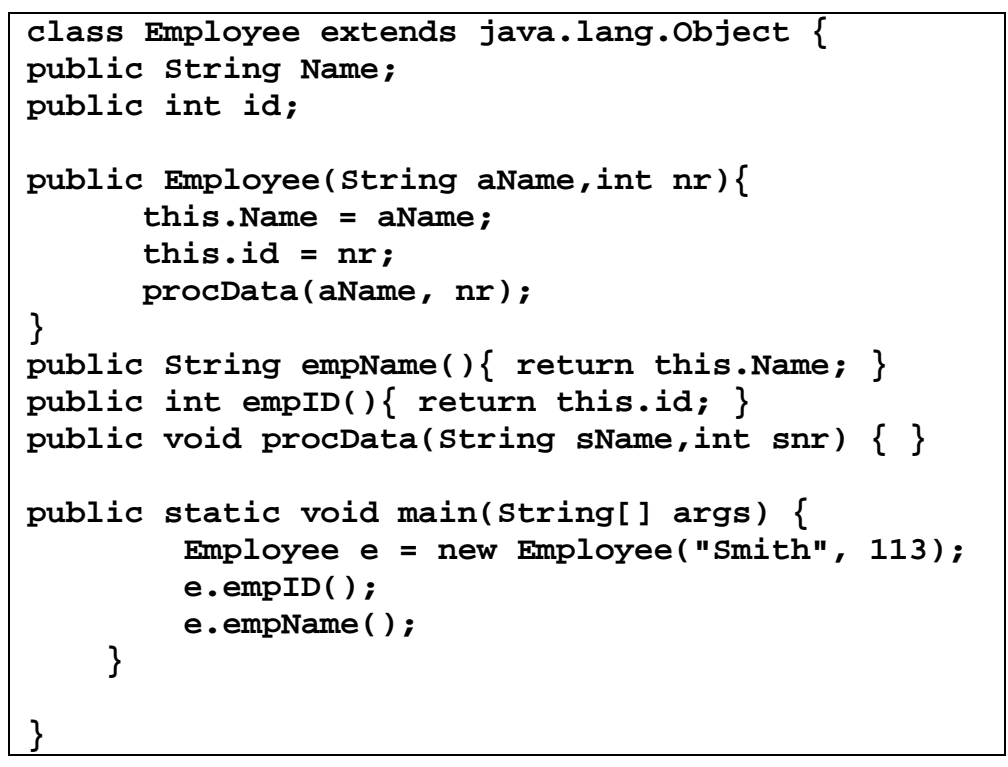

The intermediate code built by Java compiler update 22 version, for each method is using Java Development Kit (JDK), 1.5.0 presented in table 5.

Table 5. Java bytecode for Employee class

\begin{tabular}{|c|c|c|c|}
\hline Method header & $\begin{array}{l}\text { Bytecode } \\
\text { offset }\end{array}$ & $\begin{array}{l}\text { Operation } \\
\text { code }\end{array}$ & Bytecode instruction \\
\hline $\begin{array}{l}\text { public } \\
\text { Employee (java.lang. } \\
\text { String, int); }\end{array}$ & $\begin{array}{l}0 \\
1 \\
4 \\
5 \\
6 \\
9 \\
10 \\
11 \\
14 \\
15 \\
16 \\
17 \\
20\end{array}$ & $\begin{array}{lll}\text { 2A } & & \\
B 7 & \odot \odot & 01 \\
\text { 2A } & & \\
2 B & & \\
B 5 & \odot \odot & \odot 2 \\
2 A & & \\
1 C & & \\
B 5 & \odot \odot & 03 \\
2 A & & \\
2 B & & \\
1 C & & \\
B 6 & \odot \odot & 04 \\
B 1 & \end{array}$ & $\begin{array}{l}\text { aload_0 } \\
\text { invokespecial \#1; } \\
\text { //Method java/lang/Object. "<init>": ( )V } \\
\text { aload_0 } \\
\text { aload_1 } \\
\text { putfield \#2; } \\
\text { //Field Name:Ljava/lang/String; } \\
\text { aload_0 } \\
\text { iload_2 } \\
\text { putfield \#3; } \\
\text { //Field id:I } \\
\text { aload_0 } \\
\text { aload_1 } \\
\text { iload_2 } \\
\text { invokevirtual \#4;//Method } \\
\text { procData:(Ljava/lang/String; I)V } \\
\text { return }\end{array}$ \\
\hline $\begin{array}{l}\text { public } \\
\text { java. lang. String } \\
\text { empName ( ); }\end{array}$ & $\begin{array}{l}0 \\
\odot \\
1\end{array}$ & B⿺ & $\begin{array}{l}\text { aload_o } \\
\text { getfield \#2; } \\
\text { //Field Name: Ljava/lang/String; } \\
\text { areturn }\end{array}$ \\
\hline public int empID(); & $\begin{array}{l}0 \\
1 \\
4\end{array}$ & $\begin{array}{l}\text { 2A } \\
\text { B4 } \odot \odot \odot 3 \\
\text { AC }\end{array}$ & $\begin{array}{l}\text { aload_@ } \\
\text { getfield \#3; //Field id:I } \\
\text { ireturn }\end{array}$ \\
\hline $\begin{array}{l}\text { public void } \\
\text { procData(java.lang. } \\
\text { String, int); }\end{array}$ & $\theta$ & B1 & return \\
\hline $\begin{array}{l}\text { public static void } \\
\text { main(java.lang.Stri } \\
\text { ng[]); }\end{array}$ & $\begin{array}{l}0 \\
3 \\
4 \\
6\end{array}$ & $\begin{array}{lll}\text { BB } & 00 & 05 \\
59 & & \\
12 & 06 & \\
10 & 71\end{array}$ & $\begin{array}{l}\text { new \#5; //class Employee } \\
\text { dup } \\
\text { ldc \#6; //String Smith } \\
\text { bipush } 113\end{array}$ \\
\hline
\end{tabular}




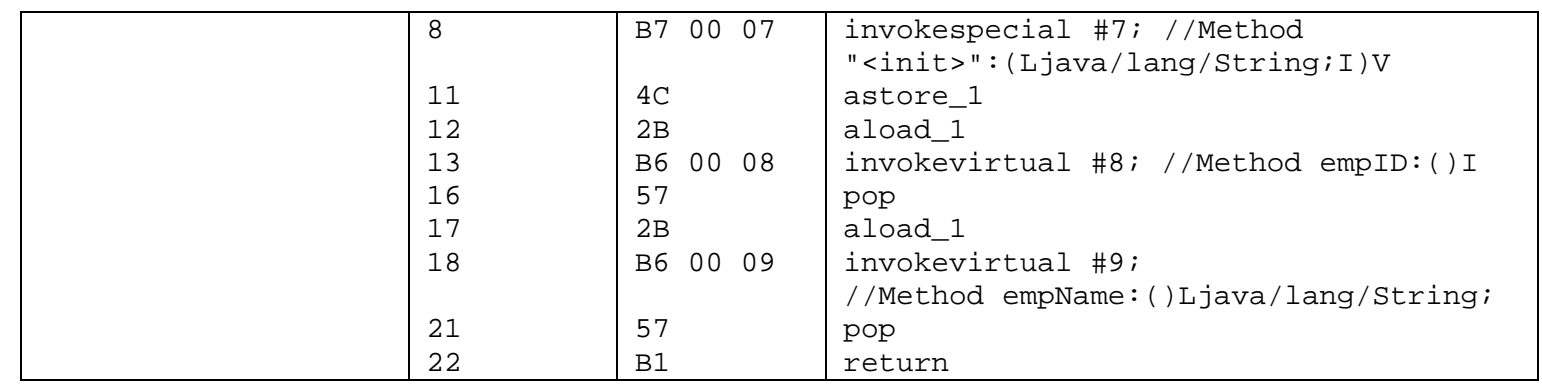

JVM identifies the data structures where the operation codes of the intermediate instructions are stored in compiled Java file. JVM has a stack-based architecture used to translate the intermediate instructions to microcontroller or microprocessor native code. More details regarding the .NET and Java virtual machines are presented in [1], [2], [4], [5], [6], [8] and [9].

\section{Binary Code Instrumentation for Malicious Software Detection}

Code instrumentation is a technique that inserts code to analyze and modify the behavior a program [3], [10]. Depending on code representation, the code instrumentation is done for:

- Source code - text representation of the program;

- Binary code - binary encoded instructions of the program.

Code instrumentation techniques are applied for the following analysis levels:

- Static analysis - the program instrumentation occurs before the program running;

- Dynamic analysis - the program behavior is analyzed by collecting runtime information.

Static binary code instrumentation. It is method of analyzing the behavior of a binary application by modifying its object code or executable code before the program is run.

Static binary code instrumentation needs advanced knowledge about the executable formats like EXE and PE. The instrumentation techniques and analysis are more expensive than the dynamic instrumentation. Two important and particular issues are distinguished during static instrumentation, according to [7]:
- The binary application is statically disassembled; the disassembled code has not accurate information about the symbols and high level information regarding the symbols;

- Instrumented code requires more space than the original binary code; moving functions from the original binary code to a new location requires relocation information regarding the entry points provided by the compiler.

Static binary code instrumentation faces with the following challenges [7]:

- Function boundaries and stack conventions - in disassembled code, functions may have multiple entry and/or exit points; the function exit may be caused by a jump instead of return instruction; using the stack may follow unusual methods for Extended Stack Pointer (ESP) and Extended Base Pointer (EBP) register handling in $\mathrm{x} 86$ 32 bit computer architecture; therefore, there is a lack of information replaced by assumption information during the binary instrumentation;

- Position-independent code - there are multiple mechanisms for position code relative addressing; static binary instrumentation involves relocating of function without relocating of static data; this issue needs fixing the position code relative accesses or avoiding relocation of the binary sequences that accesses the relative addresses;

- Scalability and modularity - code instrumentation and analysis is done on modularity principle ensuring the scalability of the binary instrumentation to large programs, keeping the accuracy and speed of the process; 
- Local variables - may be accessed in different ways: using ESP as base pointer, using computer processor general register ECX by some functions like main in $\mathrm{C}$ language, using $\mathrm{EBP}$ to access the function parameter or in general purpose;

- Actual parameters - may be passed on the stack or via registers; they may be stored at stack-relative addresses or in the top of the stack when the binary code is optimized; the number of actual parameters are not established by a local examination of the function call;

- Aliasing - multiple pointer expressions may reference the same memory, increasing difficulty to identify the instructions that accesses that memory location; pointer analysis is very difficult without high-level information about variables, array sizes, types, and so forth;

- Functions with side-effects - some operations are performed by function itself instead of its caller, affecting the content of some variables and/or registers.

Binary instrumentation is a well-known technique used in computer security to analyze binary applications in exploit detection, sandboxing, malware analysis and so forth.

Dynamic binary code instrumentation. It is a method of analyzing the behavior of a binary application when is running. The analysis consists of code injection and examination of the effects on the binary code.

The benefits of the dynamic binary code instrumentation are highlighted in [10]:

- Recompiling or relinking is not needed;

- Code discovered at run-time;

- Generated code is handled dynamically;

- It is attached to running code.
A dynamic binary code instrumentation tool is Pin. Pin allows insertion of arbitrary code into executable when the program is running. Also, Pin can be attached to a running program [10].

Pin works like a "just in time" (JIT) compiler. Pin generates binary code that is executed. The code injection is done into generated binary code, the only code ever executed. Instrumentation is not done for instructions that are ever executed, being placed into conditional branches avoided by the execution flow of the program.

Dynamic binary code instrumentation done by Pin consists of two components [10]:

- A mechanism to establish the insertion points and what code to be inserted;

- The code to be executed at insertion points.

Pin tool implements the following instrumentation techniques for analyzed executable files [10]:

- Instruction instrumentation - includes the below possible operations:

- Simple instruction counting instruments a binary program to count the number of instructions executed; Pin tool inserts a call to count function before every instruction; the value of instruction count is saved in a file called inscount.out when the executable file finishes its running; the instruction count function is very simple, using a static integer variable and is passed as argument to a function called by Pin tools before every instruction encountered in the executable file; functions are defined in inscounto.cpp from Pin tool [10];

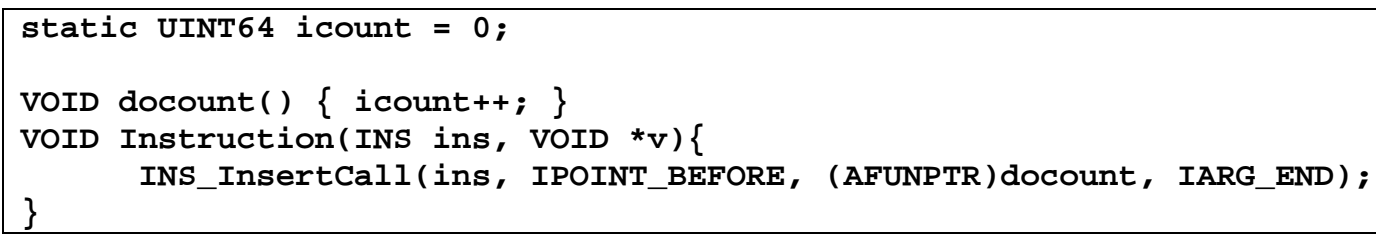


The INS_Insertcall function inserts a call to docount function relative to instruction ins. IPOINT_BEFORE is an enumerator always valid for all instructions and specifies the place of insertion. IARG_END is the argument list terminator for INS_InsertCall function.

- Instruction address tracing instruments the memory addresses where the binary instructions are stored during execution of the program; Pin tool inserts a call to trace function before every instruction in the executable file; the function writes the content of Instruction Pointer (IP) register into an output file managed by a global variable called trace in itrace.cpp source file defined in Pin tool [10];

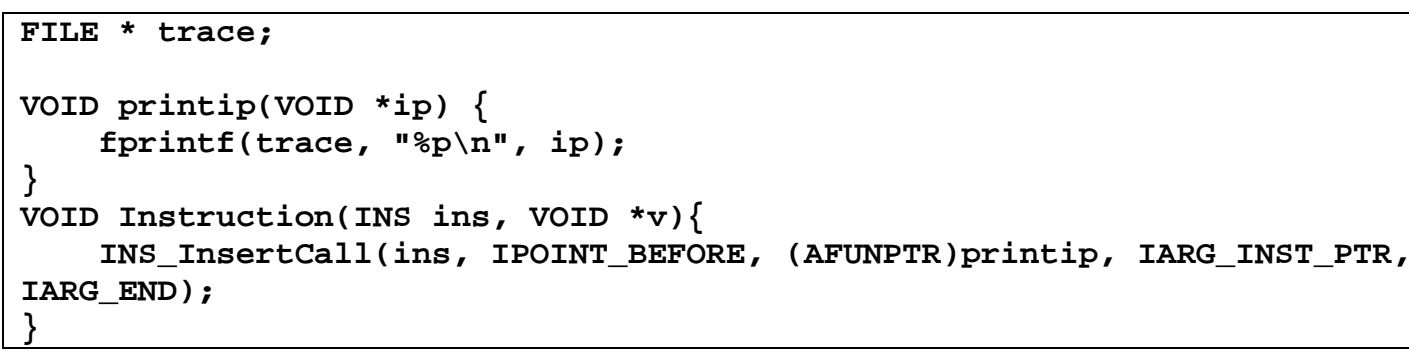

The printip function writes the content of IP register into the file managed by trace variable. The IP value is received by ip parameter and it has to be passed to INS_Insertcall function. The argument list of INS_Insertcall function is changed and the address of the instrumented instruction is passed by IARG_INST_PTR enumerator. Pin allows passing the content of different programming recipients by fields defined in the enumerator type called IARG_TYPE;
- Memory reference tracing - is a selective instrumentation technique, aiming a set of instructions from the binary code instrumented; for example, only instructions that read or write memory are considered; Pin tools inserts analysis function calls when each time when a memory operand is encountered during the execution flow of the binary program; the source code of this dynamic instrumentation technique is given in pinatrace.cpp source program and the results are saved into pinatrace.out file by the Pin tool [10];

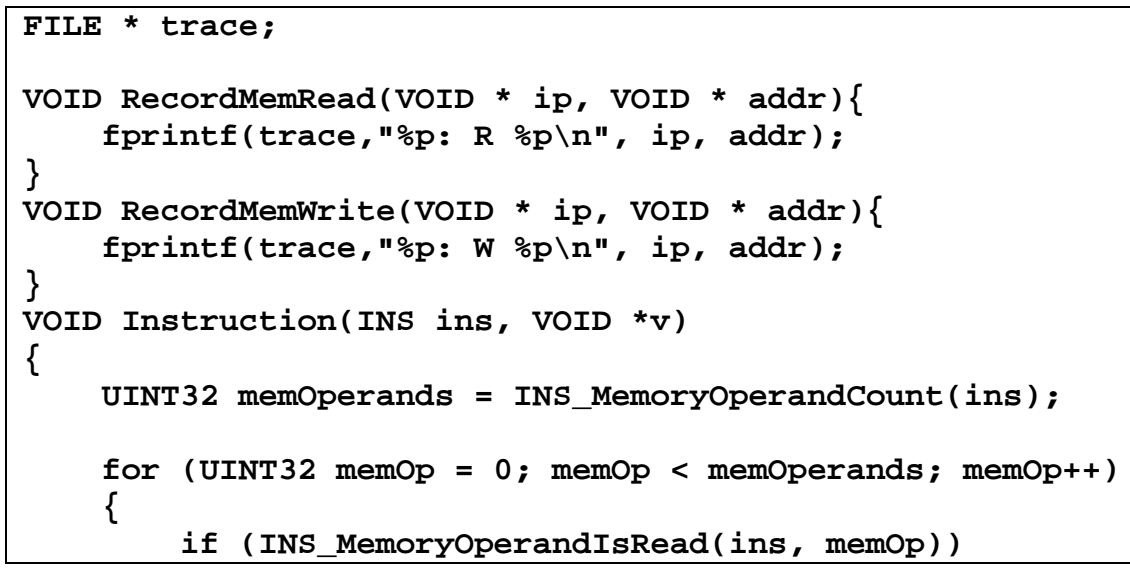




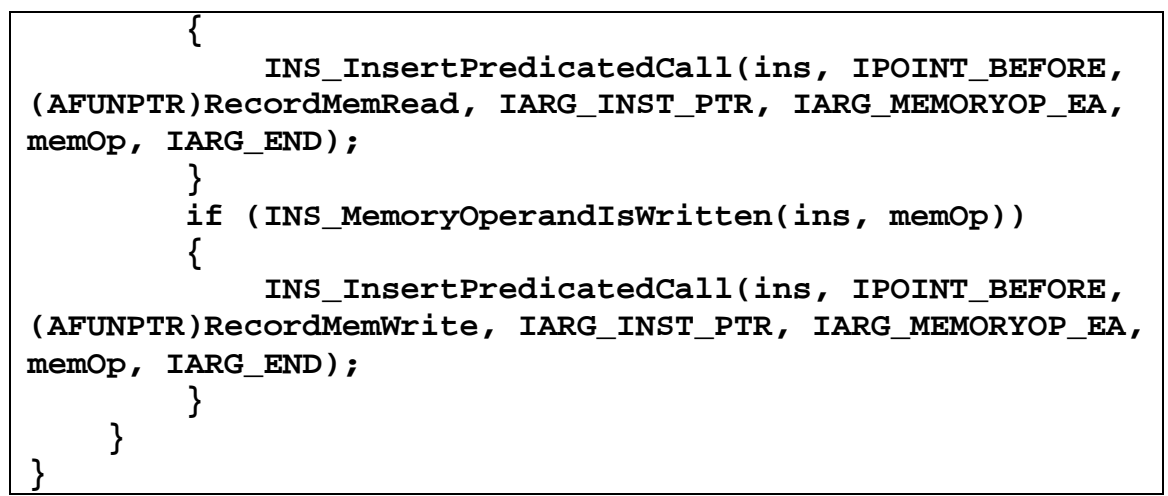

The RecordMemRead function prints a read memory record to the output file. The RecordMemWrite function prints a write memory record to the output file. There is a single output file called pinatrace.out. The memory record has the layout:

\section{(IP, memOpType, memAddr)}

where:

o IP-Instruction Pointer register, containing the address of the instruction;

o memOpType - type of the memory operation; it takes $\boldsymbol{R}$ or $W$ as values;

o memAddr-the memory address of the operand;

The Instruction function is called for each instruction, instrumenting the memory read and write operations. The number of memory operands is returned by INS_MemoryOperandCount function for each instruction ins. The memory operation type is checked by
INS_MemoryOperandIsRead

and

INS_MemoryoperandIsWritt en functions, requiring the ins instruction and memop operand index.

The

INS_InsertPredicatedCall

function avoids the predicated instructions with false predicate. The function call passes the instruction, the call place, the address of RecordMemRead or RecordMemWrite function, the instruction address, the effective address of the memory operand by IARG_MEMORYOP_EA enumerator, the operand index of the instruction, and the argument list terminator.

- Image instrumentation - aims loading and unloading of images. A trace file called imageload.out is created and contains trace messages related to loaded or unloading images. The source program containing the application for image instrumentation technique is imageload.cpp [10]. The needed functions are described below.

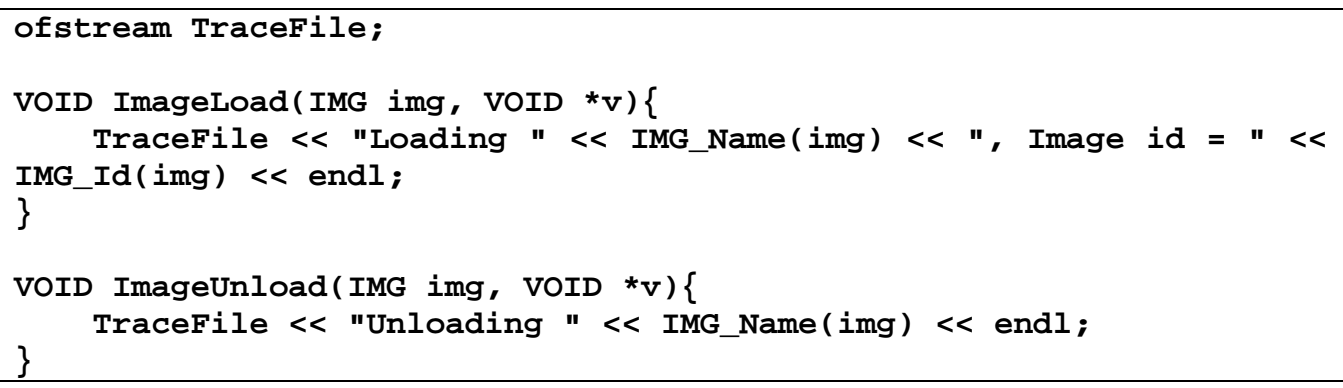


IMG_AddInstrumentFunction (ImageLoad， 0 );

IMG_AddUnloadFunction(ImageUnload， 0 ) ;

The ImageLoad function is called by Pin tool every time when an image is loaded. Also, the image can be instrumented. An image is a binary code sequence stored separately as the instrumented binary code. The ImageUnload function is called when a new image is unloaded. An image that is about to be unloaded cannot be instrumented. The IMG_AddInstrumentFunction function is called to register the ImageLoad callback to catch the loaded image. The
IMG_AddUnloadFunction function call passes the image that is about to be unloaded and the value of ImageUnload function. The unloaded images cannot be instrumented, so the ImageUnload is not an instrumentation function.

- Trace instrumentation - is a more efficient instruction counting. The counter is incremented per basic block instead of each instruction. In [10], trace instrumentation is implemented in inscount 1. cpp application.

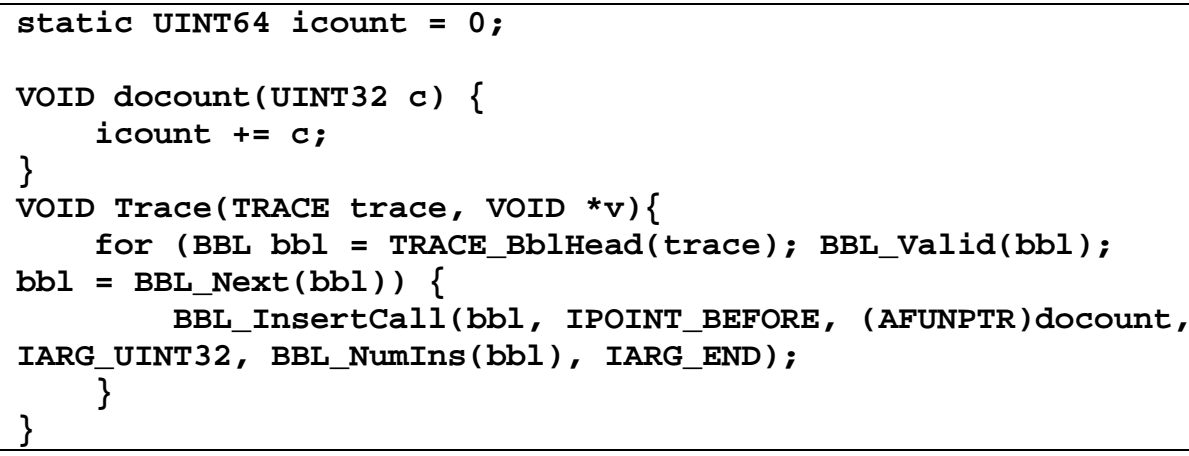

The docount function is called before every basic block. The trace parameter is an object assigned to a sequence of instructions with a single entrance and multiple exits. The for instruction parses the basic block set of the binary application and inserts a call to docount function before every basic block by BBL_InsertCall function. The BBL_InsertCall function call passes the basic block to be instrumented, the call place, the instrumentation function, the argument of docount function, number of instructions within the bbl basic block and argument list terminator.

- Routine instrumentation - is made in [10] by number of calls for each procedures and the number of instructions executed in each procedure. The instrumentation report is printed to proccount.out file, and the source file of the application is proccount . cpp.

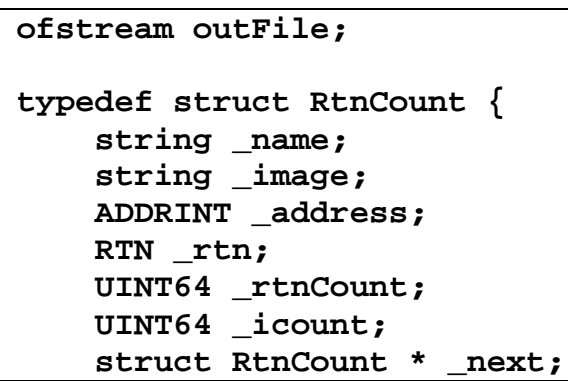




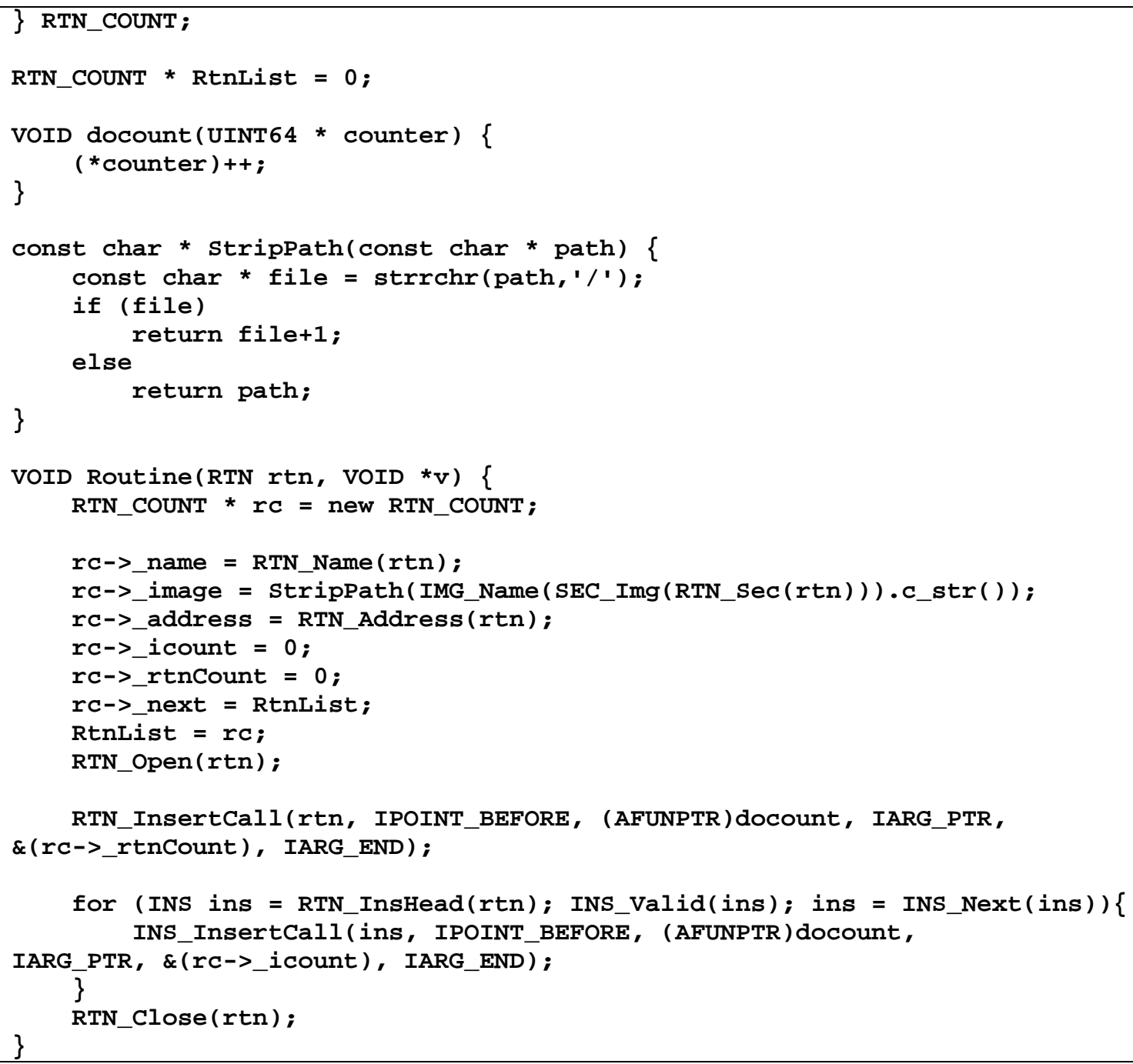

The Routine function is called every time when a new routine is executed. The rc local variable is a simple linked list to store information related to current routine. The current running routine is added to global list of routine managed by RtnList global variable. The call count of the current routine is incremented by RTN_InsertCall function call. The instruction counter for each routine is also stored by the simple linked list. The incrementing of instruction counter is made by INS_InsertCall function call. The routines are managed by routine objects and a certain routine is instrumented by referring the object name.
Binary code instrumentation provides information about the binary applications and helps to improve the correctness and speed of the developed programs. Also, the program behavior checking provides valuable information about third-party application, identifying the suspicious behaviors of the programs from unsecure sources.

\section{Methods and Techniques of Binary Code Injection}

Binary code injection inserts a binary sequence into a binary computer program to change the intended course of execution.

A way to change the course of execution in native executable files is altering of IP register. IP is a special-purpose register that stores the memory address of the next instruction to be executed. It cannot be 
programmatically accessed and its content is altered by instructions such as jmp, call and ret. These instructions permit the access to old IP values automatically pushed onto stack.

The stack content for x86-16bit computer architecture when a function is called is depicted in Figure 2.

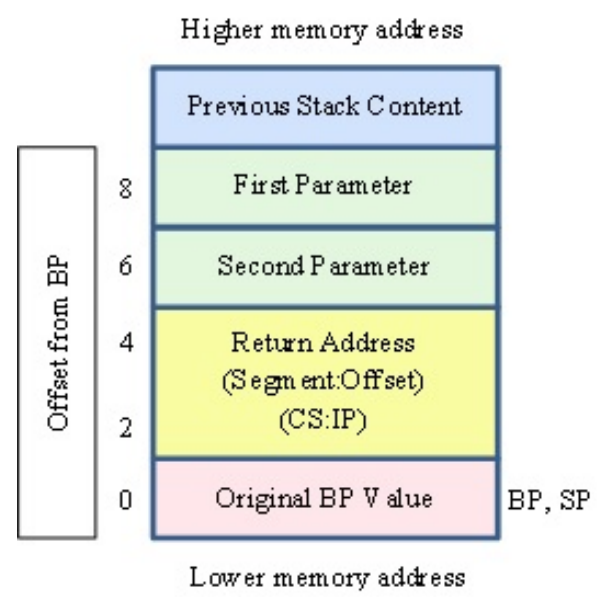

Fig. 2. Accessing stack content in a far function

The above stack content is available after a far function call with two arguments. All local variables are allocated on stack at lower memory addresses, and the return address to caller has the offsets from the current BP as 2 bytes for IP register restore and 4 bytes for CS register restore.

The IP content is restored when the ret instruction is executed. All these issues together with a stack content management may cause the change the course of execution.

To get the current value of the IP register, a dummy function is defined and its call is followed by extracting the value from the top of the stack. Therefore, the stack portion where the return address is stored may be overwritten by the extracted value of IP, and the program execution is altered.

For x86-16bit computer architecture, the register size is 16 bits. Also, the computer word has 16 bits length. For x86-32bit computer architecture, the register size and computer word are extended to 32 bits. The IP, BP and SP registers are included in the extended versions called EIP, EBP and ESP registers.

An example for IP content handling is presented below. The binary code of the sequence is injected into the binary code of the Employee constructor method presented in chapter 1 .

Table 6. Native code injection

\begin{tabular}{|c|c|c|c|}
\hline Method header & $\begin{array}{l}\text { Memory } \\
\text { address }\end{array}$ & $\begin{array}{c}\text { Binary assembly } \\
\text { instruction }\end{array}$ & Assembly instruction \\
\hline Employee $\left(\right.$ char $^{*}$, int $) ;$ & 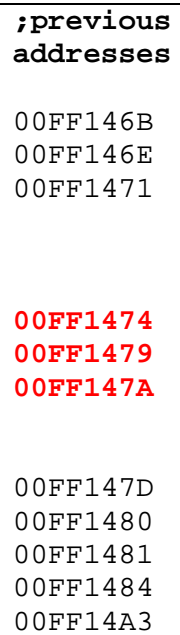 & 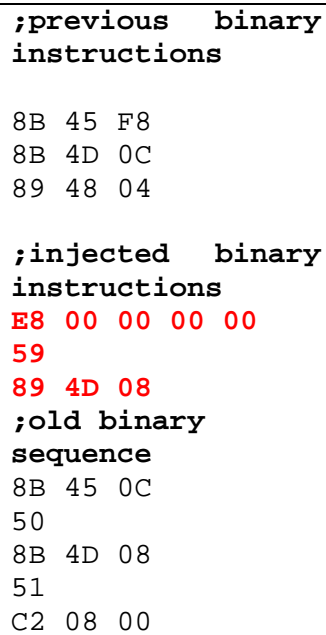 & $\begin{array}{l}\text {;previou asm instructions } \\
\text { mov eax, dword ptr [this] } \\
\text { mov ecx, dword ptr [nr] } \\
\text { mov dword ptr [eax+4], ecx } \\
\text {;injected asm instructions } \\
\text { call dummy (ofF1479h) } \\
\text { pop ecx } \\
\text { mov dword ptr [aName], ecx } \\
\text {;old asm sequence } \\
\text { mov eax, dword ptr [nr] } \\
\text { push eax } \\
\text { mov ecx, dword ptr [aName] } \\
\text { push ecx } \\
\text { ret } 8\end{array}$ \\
\hline
\end{tabular}


The effect of the injected Employee code in constructor method is a wrong aName string parameter passed to procData method. The IP content popped by ECX register is the address of the pop ecx instruction. A disastrous effect is produced when the restored IP register contains a malicious code address.

Also, elaborated stack buffer overflow attacks can be carried out by redirecting the execution flow to the shellcode that overwrites the vulnerable buffer of the target host.

The bytecode injection consists in changing the existing bytecode to redirect the execution flow built by virtual machine. For Java programming language, that means modification of the compiled file stored by the class file and interpreted by the Java Virtual Machine. That issue is available for programs with malicious intent. However, there are situations when code injection techniques are used for bytecode instrumentation of the third-party libraries which the source code is not available or a debugger or a profiler cannot be used. Also, the bytecode injection is used in performance monitoring of the application [12].

The bytecode injection allows modification of third-party bytecode without the source code availability. The injection is asked by existence of some bugs or limitations of the third-party software to see the behavior change and possible solutions to pass over the software problems. The following considerations are taken into account by [12] as good reasons to apply injection techniques:

- The exception generated by a method during a data set processing provides no information about what data element has caused it;

- Collecting performance statistics;

- The failure of a data batch processing when a data set is send to a database by Java Database Connectivity (JDBC).

Advantages of Java bytecode injection are presented in [12]:

- Modification of the binary code when the source code is not available;
- Collecting run-time information when tools like debuggers and profilers cannot be used;

- Modularity of the injected bytecode;

- Keeping the original bytecode by using tools for bytecode injection;

- Doing injection at compile-time when the bytecode is built or run-time when the target classes are loaded by the JVM.

The Java bytecode format is presented in [1], [2], [4], [5], [6], [8] and [9]. Depending on level of abstraction, the injection tools aim [12]:

- Direct bytecode manipulation (ASM tool)- Java bytecode understanding is needed because the level of abstraction is very low; the developer has to work with operation codes, the operand stack and bytecode instructions;

- Intermediate level (Javassist tool) - the code is given in strings, the classfile structure has a level of abstraction;

- Advices in Java (AspectJ tool) - the code to be injected has a syntax-checked format and it is compiled.

The injection techniques are classified by [12] into the following categories:

- Manual injection - the developer knows the place where the code is injected; to do that, the developer must know the classfile format;

- Primitive pointcuts - pointcut is an expression telling the place where a particular bytecode must be injected; this injection techniques has limitation about the place: a particular method, all public methods of a class and so forth;

- Pattern matching pointcut expressions match the target bytecode based on a number of criteria.

The bytecode injection time may be [12]:

- Manually at run-time - the bytecode asks for injected bytecode;

- Load-time - bytecode injection is performed when the target bytecode is loaded by the JVM;

- Build-time - the bytecode is modified by injection before packaging and deploying the software application. 
According to [1], [2], [4], [5], [6], [8] and [9] where the Java classfile structure is explained, injection of a new method into classfile involves the structure information modifications inside the classfile in addition to the method bytecode. Modifications are manually done in the classfile or implemented in libraries as injection tool. The operations needed to keep a viable classfile to be correctly interpreted by the JVM are:

- Addition of the method in the Constant Pool - write the information as a constant pool entry:

- Adding UTF8 method name to constant pool;

- Adding UTF8 descriptor index to constant pool;

- Adding method name type to constant pool;

- Adding method type to constant pool;

- Injection of the method bytecode inserting the method binary code into the classfile:

- Insertion of the method bytecode;

- Write the bytecode image;

- Adjusting the offsets: code length, maximum stack, exception table, code attributes, and attribute length.

The new content of the classfile has to meet the structural constraints to pass the verification proceeded by JVM in order to interpret the bytecode correctly.

\section{Conclusions}

Binary code instrumentation offers the tools needed to increase the security and reliability of a software application. Therefore, the developers are able to understand how an exploit is created to pass the security mechanisms of the application in order to build defense techniques.

Also, the binary code instrumentation may be used reverse engineering of malwares in order to detect them and implement protection techniques.

The system security depends on the safety of running binary code, operating system kernel internals, linker and loader internals that have to be known and understood by software developers.

\section{References}

[1] C. Boja and M. Doinea, "Security Assessment of Web Based Distributed Applications", Informatica Economică, vol. 14, no. 1, 2010, pp. 152 - 162

[2] A Danehkar, "Inject your code to a Portable Executable File", 27 December 2005, http://www.codeproject.com [Nov. $15,2013]$

[3] F. Falcon and N. Riva, "Dynamic Binary Instrumentation Frameworks: I Know You're there Spying on Me", Core Security, June 2012

[4] R. Paleari, "Static disassembly and analysis of malicious code", 5 July 2007, http://roberto.greyhats.it/talks.html [Nov. 15, 2013]

[5] M. Pietrek, "An In-Depth Look into the Win32 Portable Executable File Format", MSDN Magazine, http://msdn.microsoft.com /enus/magazine/cc301805.aspx [Nov. 15, 2013]

[6] M. Popa, "Binary Code Disassembly for Reverse Engineering", Journal of Mobile, Embedded and Distributed Systems, vol. 4, no. 4, 2012, pp. 233 - 248,

[7] P. Saxena, R. Sekar and V. Puranik, "Efficient Fine-Grained Binary Instrumentation with Applications to Taint-Tracking", http://seclab.cs.stonybrook.edu/seclab/pu bs/cgo065-saxena.pdf [Nov. 15, 2013]

[8] C. Toma, "Sample Development on Java Smart-Card Electronic Wallet Application", Journal of Mobile, Embedded and Distributed Systems, vol. 1, no. 2, 2009, pp. $60-80$

[9] G. Vigna, "Static Disassembly and Code Analysis, Malware Detection. Advances in Information Security", Springer, Heidelberg, vol. 35, 2007, pp. $19-42$

[10] Pin 2.13 User Guide, Intel Developer Zone, http://software.intel.com/sites/ landingpage/pintool/docs/61206/Pin/html / [Nov. 15, 2013] 


2010_08_01_archive.html [Nov. 15,
2013]
$[12] \quad \begin{aligned} & \text { 2011/09/07/practical-introduction-into- } \\ & \text { code-injection-with-aspectj-javassist-and- } \\ & \text { java-proxy/ [Nov. 15, 2013] }\end{aligned}$

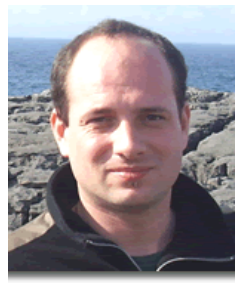

Marius POPA has graduated the Faculty of Cybernetics, Statistics and Economic Informatics in 2002. He holds a $\mathrm{PhD}$ diploma in Economic Cybernetics and Statistics. He joined the staff of Academy of Economic Studies, teaching assistant in 2002. Currently, he is Associate Professor in Economic Informatics field and branches within Department of Economic Informatics and Cybernetics at Faculty of Cybernetics, Statistics and Economic Informatics from Bucharest University of Economic Studies. He is the author and co-author of 9 books and over 140 articles in journals and proceedings of national and international conferences, symposiums, workshops in the fields of data quality, software quality, informatics security, collaborative information systems, IT project management, software engineering.

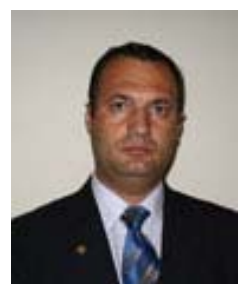

Sergiu CAPISIZU has graduated the Faculty of Cybernetics, Statistics and Economic Informatics in 1997 and National University of Defense in 2005. He holds a PhD diploma in Economic Cybernetics and Statistics, having the title Models and techniques to perform the economic information audit. He is co-author of books and articles in information audit and ICT fields. Also, he has published articles in proceedings of national and international conferences, symposiums, workshops in the fields of data quality, software quality, information audit and juridical aspects in ICT field. He is evaluator of ANEVAR association. 